\title{
"LA FORTALEZA DE ARBECA. EL PROYECTO VILARS 2000". INVESTIGACIÓN, RECUPERACIÓN Y SOCIALIZACIÓN DEL CONOCIMIENTO Y DEL PATRIMONIO
}

\author{
“LA FORTERESSE DE ARBECA. LE PROJET VILARS 2000”. RECHERCHE, \\ RECUPERATION ET SOCIALISATION DE LA CONNAISSANCE ET DU PATRIMONIE
}

\author{
NATÀLIA ALONSO (*) \\ EMILI JUNYENT $(*)$ \\ ÁNGEL LAFUENTE (*) \\ JOAN B. LÓPEZ $(*)$ \\ ENRIC TARTERA $(*)$
}

\section{RESUMEN}

La fortaleza de Els Vilars (Arbeca, Lleida), construída en torno al 750 cal. ANE y abandonada poco después del 350ANE, constituye un yacimiento clave para la comprensión de la Primera Edad del Hierro y la Época Ibérica en sus fases antigua y plena en el noreste de la Península Ibérica. Sus defensas excepcionales, muralla torreada, barrera de piedras hincadas y foso, la convierten en referente de la fortificación ibérica. El proyecto "Vilars 2000" tiene por objeto la investigación, la recuperación patrimonial y la difusión pública. Se exponen los objetivos del Plan Director, así como la estrategia de obtención de recursos económicos, incidiendo en su condición de valor turístico de primer orden.

\section{RÉSUMÉ}

La forteresse de "Els Vilars" (Arbeca, Lleida), bâtie vers 775 cal. BC et abandonnée un peu après l'année 350 $B C$, devient un site clé pour l'étude du premier Âge du Fer et la période ibérique ancienne et pleine dans le nord-est de la Péninsule Ibérique. Ses défenses exceptionnelles, rempart flanqué de 12 tours, chevaux-de-frise et fossé, permettent la considérer comme le point de référence des fortifications ibériques. Le projet "Vilars 2000" a comme buts la recherche scientifique, la récupération patrimoniale et la

(*) Grup d'Investigació Prehistòrica. Unitat d'Arqueologia, Prehistòria i Història Antiga. Universitat de Lleida. Pl. Víctor Siurana, 1.25003 Lleida. Correo electrónico: arqueologia@ historia.udl.es ejunyent@historia.udl.es

El artículo fue remitido en su versión final el 30-X-2000. diffusion au grand public. Dans cet article sont présentés les objectifs du Plan Directeur, ainsi que la stratégie pour obtenir les moyens économiques nécessaires, compte tenu de l'intérêt touristique majeur du site.

Palabras clave: Arqueología. Primera Edad del Hierro. Grupo del Segre-Cinca. Iberos. Ilergetas. Fortificación. Musealización. Rutas arqueológicas. Internet. Realidad Virtual.Video.

Mots clé: Archéologie. Premier Âge du Fer. Groupe du Segre-Cinca. Ibères. Ilergètes. Fortification. Traitement muséographique. Itinéraires archéologiques. Internet. Restitution virtuelle. Video.

\section{LA INVESTIGACIÓN}

La fortaleza de Els Vilars de Arbeca constituye una de las piezas claves en el marco general de un proyecto de investigación, "Un modelo singular de la transformación de las sociedades segmentarias en sociedades complejas: el Valle del Segre (II y I milenio ANE)" (DGES, MEC, PB96-0419), a su vez heredero de otro anterior "Primera Edad del Hierro y Época Ibérica en la Catalunya Occidental. Territorio y urbanismo" (DGICYT, PS-0148), y del trabajo de un equipo, el Grup d'Investigació Prehistòrica de la Universitat de Lleida.

Dicho proyecto tiene por objetivo caracterizar en las llanadas occidentales catalanas ese proceso histórico que, a caballo del segundo y el primer mile- 


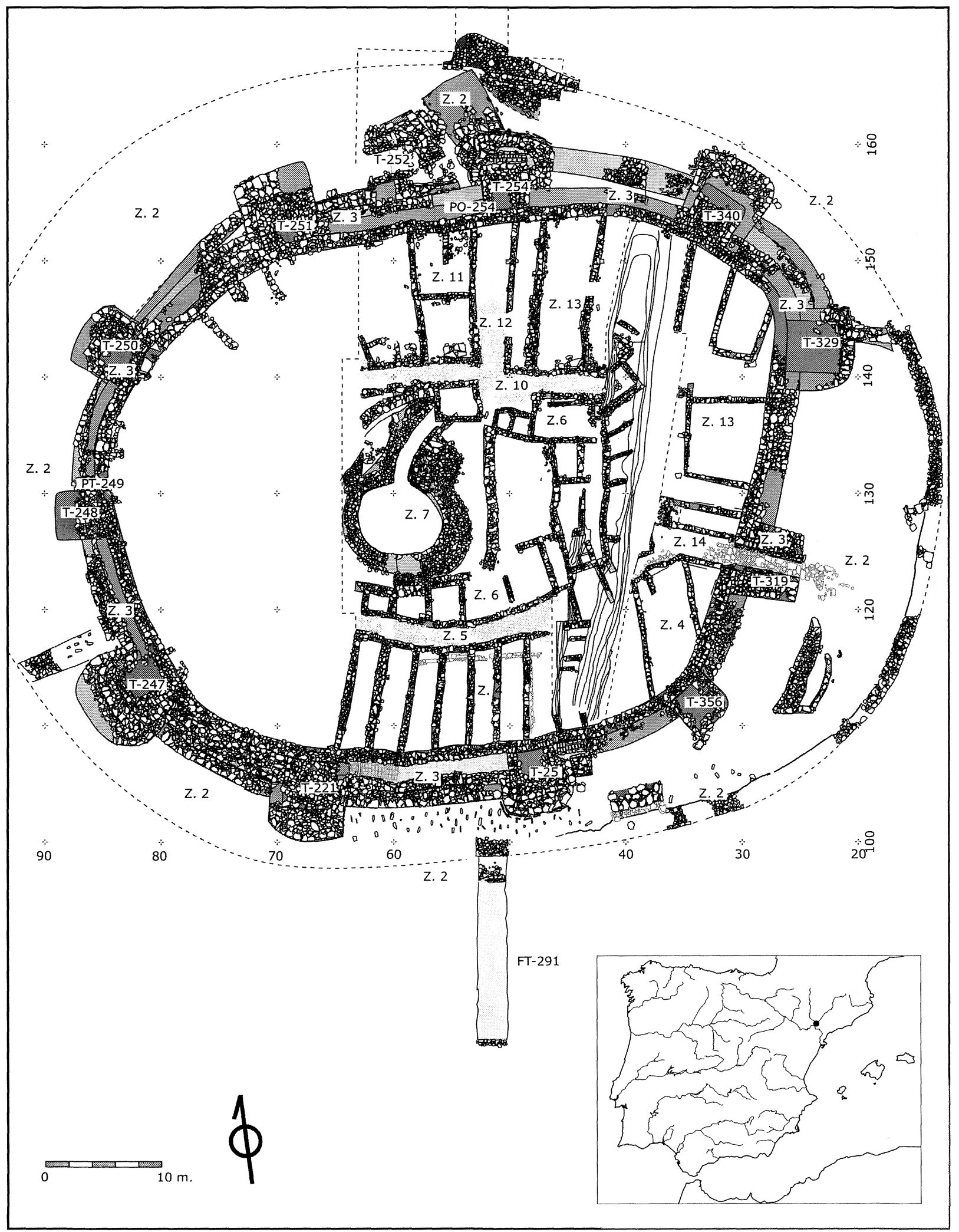

Fig. 1. Localización en la Península Ibérica de la fortaleza de Els Vilars (Arbeca, Lleida) y planta general de la misma.

T. P., 57, n. $^{\circ} 2,2000$ 
nio, lleva a la aldea a convertirse en ciuitas. Desde la aparición de las grandes aldeas de patrón disperso tipo Minferri (Juneda) a la definición del Grupo del Segre-Cinca, la formación social aldeana y sus pequeños poblados cerrados, ejemplificados por Genó (Aitona); desde ésta a la aparición de jefaturas y linajes aristocráticos y, más tarde, al desarrollo de la etnia ilergeta como una sociedad estatal arcaica, politribal, con un territorio extenso y definido, presidido por la ciuitas y el regulus.

El proyecto se propone, también, mostrar la singularidad de este proceso histórico y de su materialización concreta en el registro arqueológico en relación con el noreste peninsular estricto, es decir, la costa catalana, y el sur de Francia por debajo del Hérault. Una de las claves explicativas de esta originalidad, que lleva a las comunidades agrícolas del Valle del Segre a desarrollar nuevas estrategias de ocupación del territorio, un temprano urbanismo y la arquitectura en piedra, radica en la adopción y desarrollo de nuevas formas de producción agrícola. Dos tesis doctorales se han consagrado al análisis y contrastación arqueológica de dicho proceso histórico (Alonso, 1999; López, 2000).

La fortaleza de Arbeca refleja en profundidad las dimensiones fundamentales de esas transformaciones en su etapa final: nuevas estrategias de explotación y control del territorio, desarrollo de jefaturas complejas durante la Primera Edad del Hierro y tránsito al estado arcaico ilergeta. Parece obvio suponer que, a lo largo de cuatrocientos años de vida, la fortificación no desempeñó el mismo papel, sino que su suerte, su creación y su desaparición, estuvo profundamente imbricada en los profundos cambios económicos, sociales y políticos que tuvieron lugar entre el 750 y el 350ANE. La sociedad ilergeta de fines del sigloVI difería de la sociedad preibérica de la segunda mitad delVIII, pero aún eran más significativas las diferencias entre el mundo ibérico pleno de mediados del siglo IV y aquélla.

La fortaleza, nacida a mediados de la octava centuria como manifestación arrogante de fuerza en su condición de residencia de un linaje aristocrático, exaltaba su poder y prestigio sobre el territorio y las comunidades que lo ocupaban sobredimensionando sus defensas, más allá de las necesidades estrictamente militares (Fig. 1). Cuando se consolidaron formas políticas más amplias y estables, posiblemente la vieja fortaleza perdió su razón de ser. Hemos sugerido que su abandono fué provocado por la hipertrofia del sistema defensivo (Alonso et alii, 1998). Además, el reducido espacio interior resul- taba a todas luces insuficiente para las exigencias del urbanismo y la arquitectura ilergetes de la segunda mitad del siglo IV y las murallas y el foso constituían un obstáculo insalvable que impedía el crecimiento a extramuros, solución que había sido adoptada en el oppidum del Molí d'Espígol (Tornabous, Lleida). La afirmación de un nuevo marco político más amplio y estable, el del estado aristocrático ilergeta, convirtió en obsoleta aquélla expresión de las viejas jefaturas, lo que unido a las dimensiones de un recinto en el que apenas cabían hacinadas un centenar y medio de personas, significó su suerte irremediable: abandono y ruina.

"Vilars 2000", como proyecto interdisciplinar que tiene por objetivo la investigación integral de un asentamiento y su territorio, constituye hoy uno de los escasos ejemplos activos en el depauperado panorama de la arqueología ibérica catalana. El proyecto se desarrolla en una serie de líneas de investigación que encauzan las diferentes perspectivas y objetivos del equipo. Éstas se ocupan de campos imbricados entre sí y no constituyen una jerarquía de objetivos. La bibliografía producida es ya notable y puede consultarse en los últimos trabajos (Agustíet alii, 2000: 305-324;Alonso et alii, 1996: 319-339; 1998: 355-372; 1999: 287-292; 2000, 225-231; Garcés et alii, 1997; 2000: 147157).

\section{LA SECUENCIA: CRONOLOGÍA Y PRESENTACIÓN POR FASES}

La periodización del yacimiento, especialmente en sus fases más antiguas, no ha sido concebida como un andamiaje cronológico construído a partir de "fósiles directores", que tan sólo hubiera conducido a reproducir períodos culturales preexistentes. Se ha intentado establecer una temporalidad calendárica, independiente de las propuetas arqueológicas conocidas y basada en tres presupuestos teórico-metodológicos fundamentales:

1. La inserción de la secuencia de la fortaleza dentro del proceso histórico singular que se desarrolla en el valle del Segre.

2. El respeto más riguroso a la estratigrafía, los contextos arqueológicos y las actividades antrópicas inherentes para establecer la secuencia interna de la fortaleza.

3. La utilización de la calibración de las dataciones como soporte esencial para establecer la temporalidad absoluta de cada fase o período. 
En síntesis y por lo que concierne a la periodización de las diferentes fases a partir de series más o menos amplias de dataciones, nuestra propuesta se basa en la utilización de los valores centrales que proporciona la mediana de los intervalos de máxima probabilidad (IMP) de dichas dataciones calibradas a 2 sigmas, depurados hasta un mínimo del 90\% de fiabilidad (Agustí et alii, 2000: 305-324; López, 2000). Este método se compagina o contrasta con la obtención "artificial" de medias ponderadas puntuales, cuando se trata de fechar acontecimientos concretos o actividades antrópicas que cuentan con varias dataciones radiocarbónicas.

Por lo que respecta a la estratigrafía interna del yacimiento, se distinguen dos grandes horizontes culturales, materializados por diferentes refacciones urbanísticas que en ningún momento alteran el perímetro original de la fortaleza:

\section{Primera Edad del Hierro o GSC-IV: Vilars 0 y I}

Comprende la fundación ex novo de la fortaleza (Vilars 0) y una primera reconstrucción de las viviendas (Vilars I), que cambian significativamente su planta y dimensiones, reduciendo su tamaño hasta la mitad y perdiendo el vestíbulo. Desde el momento inicial, el asentamento está protegido por una muralla y once torres, un campo frisio y un foso, siendo los dos primeros elementos paulatinamente reforzados con paramentos múltiples. El recinto dispone de dos entradas: una torre-puerta al Este y una poterna al Oeste.

\section{Período ibérico: Vilars II, III y IV}

El ibérico antiguo corresponde a la fase Vilars II. No se modifica el perímetro, ni la disposición urbanística original, aunque sí parcialmente el espacio central y el trazado de algunas calles relacionadas con los accesos. Se anula la poterna Oeste y se abre una nueva puerta de entrada orientada al norte, al amparo de una de las torres antiguas y de una nueva, hueca, construída en posición adelantada. Las viviendas son ahora más complejas y compartimentadas; el foso y la barrera de piedras hincadas se han ido colmatando de tierras paulatinamente $y$, a finales del período, debían estar prácticamente fuera de servicio.

Durante el ibérico pleno (Vilars III y Vilars IV) tiene lugar, a inicios de la fase Vilars III, una importante remodelación urbanística que afecta a la distribución y orientación general de los espacios de circulación. Se construye el gran pozo o cisterna central con un bajador o corredor de acceso. En el exterior, se excava un gran foso a expensas del anterior, forrando sus taludes con un nuevo muro. Con toda probabilidad la antigua torre-puerta abierta al Este ha sido anulada; no así la puerta norte, según se desprende de las potentes estructuras que, ante ella, doblan el paramento del foso. Vilars IV se refiere a la ocupación que precede al abandono. Se trata de una fase mal conocida y peor conservada a la que se relacionan pequeñas refacciones domésticas y la obliteración intencionada de la cisterna.

En términos de cronología absoluta, ya hemos argumentado en un trabajo anterior (Agustí et alii, 2000: 305-324) que la fecha fundacional del yacimiento podría situarse en torno al $762 \mathrm{cal}$. ANE a partir de la mediana de los intervalos de máxima probabilidad (IMP) (Fig. 2, Diagrama A). Hemos insistido también en que dicha cronología podría ser incluso ligeramente más alta si tenemos en cuenta que la serie de cuatro dataciones disponible para la fase Vilars 0 cuenta con una calibración (Beta-92277) inmersa de lleno en el tramo conocido como la "catástrofe del Hierro" (Baillie y Pilcher, 1983: 58).

Habida cuenta del escaso número de dataciones todavía disponible, dicha calibración distorsiona considerablemente el valor central final, por lo cual consideramos prudente realizar otras aproximaciones sin incluirla. Así, la mediana de los IMP resultante (Fig. 2, Diagrama B) se situaría hacia el 793 cal.ANE. De la misma manera, la calibración de la media ponderada (2622 $\pm 34 \mathrm{BP})$ de dichas fechas, abogaría por una datación alta: 824 (801) 777 cal. ANE, cuyo valor central de los IMP se situaría hacia el $800 \mathrm{cal}$. ANE.

Esta relativa mayor antigüedad de la fase fundacional viene avalada también por las dataciones obtenidas durante la campaña de 1999 , referidas a la fase posterior (Vilars I), inéditas hasta hoy. Se han obtenido por AMS, a partir de los huesos de dos fetos de caballo aparecidos en el estrato de construcción (UE 4642) que precede al pavimento más antiguo de la fase Vilars I, con lo cual marcan también el final de Vilars 0.

Se trata de dos deposiciones independientes, posiblemente rituales y aparecidas en puntos diferentes de la habitación. Aún así, su presencia en un mismo estrato, que por otro lado responde a una única actividad antrópica, permite considerarlas relativamente contemporáneas o muy poco distanciadas en el tiempo. La mediana de los IMP resultante (Fig. 2) las sitúa hacia el 780 cal. ANE. 


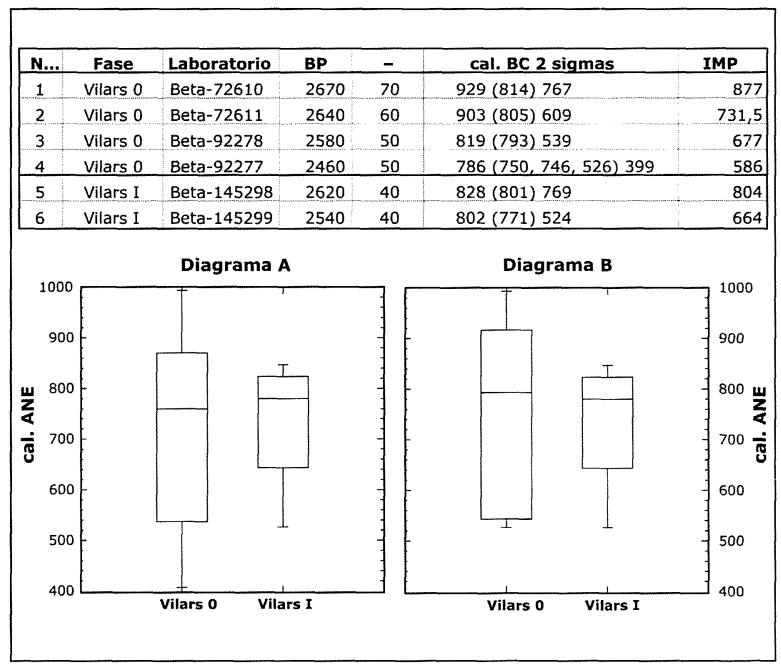

Fig. 2. Els Vilars (Arbeca, Lleida). Tabla general de dataciones y diagramas de calibración.

Este valor debe tomarse también, sin embargo, con extrema prudencia ya que la serie es muy reducida y una aproximación estadística diferente rebaja considerablemente dicha cronología, hecho que parece más coherente si se considera su posición dentro de la secuencia estratigráfica. Así, su análisis estadístico soporta perfectamente el test de " $\mathrm{t}$ " $(1,4)$ para poder ser tratadas conjuntamente, con lo cual se obtiene una media ponderada de $2579 \pm 28$ $\mathrm{BP}$, cuya calibración proporciona un valor central de los IMP en torno al $701 \mathrm{cal}$. BC.

Será necesario pues confirmar en el futuro con nuevas dataciones esta tendencia a la baja, en principio lógica, para poder establecer definitivamente en años calendáricos la secuencia interna de la Primera Edad del Hierro. A pesar de todo y teniendo presentes las limitaciones mencionadas, puede proponerse una cronología para Vilars 0 en torno al $800 / 775$ - 700/675 cal. ANE y 700/675 cal. ANE $550 \mathrm{ANE}$, para la fase Vilars I.

En efecto, el resto de la secuencia descansa sobre indicadores crono-culturales convencionales, estratificados en el propio asentamiento o fechados en contextos ajenos al mismo. La fechación atribuída a la aparición de las primeras cerámicas torneadas paleoibéricas de origen meridional o costero e ibéricas pintadas ya locales en la Cataluña occidental y el Valle del Segre, nos permiten situar vagamente en la segunda mitad del siglo VI el tránsito entre Vilars I y Vilars II. Por su parte, la cerámica gris monocroma, característica de este último, con su elemento más representativo en el plato de bor- de à marli, forma de incontestable origen grecooriental fechada entre mediados del VI y primera mitad del sigloV, nos lleva a alargar la etapa ibérica antigua hasta el tercer cuarto del siglo V.

La fechación de las fases más recientes, Vilars III y Vilars IV, viene condicionada por diferentes factores. De entrada, los niveles correspondientes han sido casi totalmente arrasados y tan sólo se conservan en el gran testigo (Zonas 0 y 8) y, por otro lado, la estrategia de excavación primando la delimitación del recinto ha "olvidado" de momento este reducido espacio interior. No se ha planteado hasta ahora la fechación radiométrica de estas fases y su cronología descansa, como decimos, sobre algunos conjuntos estratificados y materiales aislados con datación tipológica bastante precisa. Nos referimos a la presencia de figuras rojas y barniz negro ático.Vilars III se fecha a partir de unakylix-skyphos de figuras rojas próxima a los círculos del pintor de $p h i$, datable en el primer tercio de la cuarta centuria y a una kylix de pie bajodelicatte class, atribuible al último cuarto de la quinta centuria o inicios de la siguiente (Garcés y Junyent, 1988: 106; 1989, 48; Garcés et alii, 1991: 183-184).

Las cerámicas áticas de barniz negro más tardías ofrecen un terminus post quem al momento de abandono de la fortaleza. Es de destacar la inexistencia en el asentamiento de producciones atribuibles a la segunda mitad del siglo IV, razón por la cual lo hemos situado en torno a 325 ANE.

\section{LA DOCUMENTACIÓN: EL SISTEMA GENERAL DE REGISTRO}

El sistema de excavación y registro aplicado en Els Vilars contó bien pronto con la informática (Garcés et alii, 1991: 189-210). A los grandes capítulos habituales del Sistema General de Registro: Registro Estratigráfico (ficheros de información estratigráfica), Registro Documental (ficheros de fotografías, plantas, secciones y alzados), Registro de Materiales (ficheros de inventario y cuantificación de materiales y muestras) y Diagrama Estratigráfico, hemos incorporado otro nuevo: Registro Patrimonial.

\section{Patrimonio Arqueológico Vilars}

Desde la campaña de restauración y consolidación en la cara occidental del gran corte-testigo 
efectuada el año 1993, la complejidad de la intervención, la consciencia del salto cualitativo que suponía la perspectiva de recuperar la totalidad del monumento y el rigor en la documentación de cuanto se hacía, se convirtió en uno de los criterios básicos de intervención.

A partir de entonces la actuación sobre el patrimonio fue igualmente informatizada. Se incorporó al sistema de registro un nuevo fichero, PATRIAVIL, Patrimonio Arqueológico dels Vilars, que consiste en una base de datos que recoge sistemáticamente, a partir de fotografías digitalizadas, la intervención sobre cada unidad estratigráfica constructiva intervenida. Generalmente, se trata de elevaciones de muros y la ficha correspondiente recoge, mediante cuatro fotografías, su estado antes y después de la intervención, indicando las restituciones y añadidos, y recogiendo la información estratigráfica y topográfica de la U.E., el hecho arqueológico al que pertenece, datación TPQ/TAQ, fase a la que corresponde, descripción del trabajo realizado, observaciones y fecha.

\section{"Vilars Virtual"}

A partir del año 1996 el equipo se propuso otro salto cualitativo incorporando la restitución en 3D al registro y la utilización del tiempo real y los recursos multimedia en la reconstrucción global de la fortaleza: nacía así "Vilars Virtual” (Lám. I).

"Vilars Virtual" es un proyecto compartido por arqueólogos/as e informáticos/as cuyo objetivo es la creación de un modelo tridimensional que restituya el urbanismo y la arquitectura de la fortaleza, así como el paisaje de su entorno, paleotopografía y paleovegetación natural y cultivos. "Vilars Virtual" es un proyecto común del Grup d'Investigació Prehistòrica y del Grup de Recerca Interacció Humà-Ordinador del Departament de Informàtica i Enginyeria Industrial de la Universitat de Lleida.

Pretendemos desarrollar un instrumento que tiene que ser tan útil para la investigación como para la difusión social de los resultados de la investigación arqueológica. "Vilars Virtual" es una herramienta de difusión ciudadana. La restitución volumétrica y de ambientes es esencial para mostrar al público el valor patrimonial del conjunto y los resultados de la investigación. "Vilars Virtual" recoge lo esencial del doble reto del proyecto "Vilars 2000": alcanzar los objetivos científicos y socializarlos. La necesidad de optimizar la explotación de

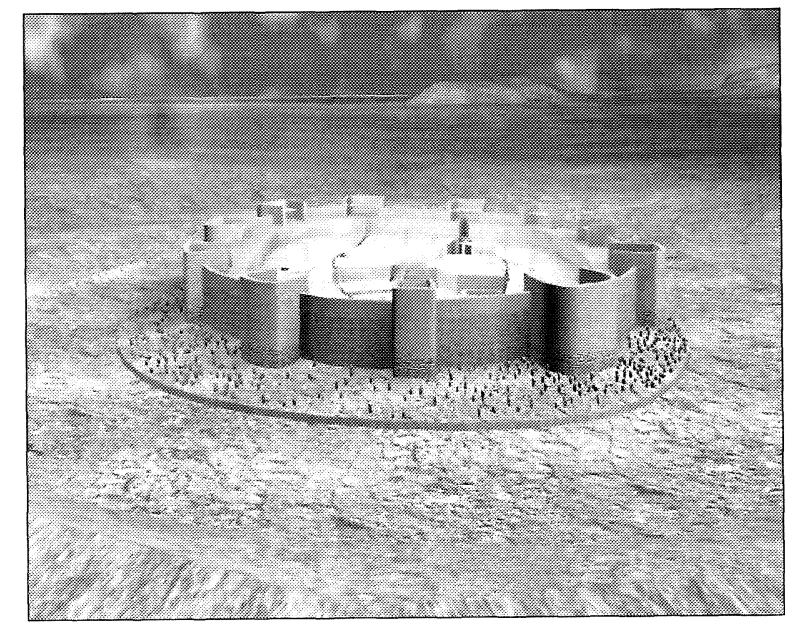

Lám. I. Representación tridimensional de la fase Vilars 0 de Els Vilars (Arbeca, Lleida).

los datos de las excavaciones y las posibilidades que representan las nuevas tecnologías para su aplicación en el campo de la arqueología justifican sobradamente los esfuerzos.

La documentación arqueológica describe los restos tal como los recuperamos. Ahora, mediante la infografía y los sistemas multimedia, pretendemos acercarnos al pasado tal como era a través del pasado interpretado. El objetivo es crear una base de datos capaz de representar de manera automática las estructuras recuperadas tal y como las interpretamos gracias a un diccionario de objetos. Pretendemos que este sistema permita una actualización constante de la base documental, ofreciendo siempre un estado de la cuestión. Trabajamos para lograr una total elasticidad en las demandas de visualización, hecho que hará posible mostrar estructuras estrictamente sincrónicas o limitar la representación espacialmente (Alonso et alii, 2000: 225-231).

Conscientes de que una imagen puede engañar más que mil palabras, nos planteamos como irrenunciable la conexión entre documentación y restitución, entre la realidad virtual y la virtualidad, para evitar la "tentación de los efectos especiales", ciñendo la reconstrucción a hechos. La realidad virtual no debe substituir a la realidad materializada en el registro sino que debe desarrollarla. No se trata de renunciar a las posibilidades de la infografía sino de utilizarla para transmitir información rigurosa, tanto cuando se pretende la simulación mimética y exacta como cuando el objetivo es una recreación virtual sin referente preciso en el registro arqueológico. 
Por eso, la propuesta de restitución deberá ser siempre reversible, haciendo posible el retorno a la información de base, y transparente, en el sentido de permitir una lectura crítica al ofrecer una visualización diferenciada de los restos recuperados, los restos restaurados y las hipótesis reconstructivas.

Un primer vídeo ha servido de prueba piloto, convirtiéndose a la vez en un instrumento de promoción de "Vilars 2000". Pese a tener poco que ver con los objetivos finales planteados, su calidad ha permitido presentarlo en el26th International Congress $C A A$, en la Muestra de RealidadVirtual celebrada en Barcelona, marzo de 1998, y en "Archeo Virtua", ler. Festival International du Multimedia pour l'Archéologie, Archéodrome de Bourgogne, marzo de 1999.

Los medios utilizados hasta la fecha son los propios de los dos equipos. El registro arqueológico documental y gráfico en 2D utiliza el entornoApple Macintosh, es informatizado en bases de datos Claris File Maker y la documentación gráfica en Adobe Illustrator. Para confeccionar el modelo virtual se partió de una planta interpretativa también realizada en Illustrator y se le dió volumen con el programa 3D Studio Max. Las texturas fueron creadas en Adobe Photoshop, a veces partiendo de dibujos vectoriales Illustrator. El montaje del vídeo se realizó con el programa Adobe Première. Tanto el modelado como el rendering y el montaje de vídeo se hicieron en sistemas compatibles IBM con Microsoft Windows 95.

\section{LA RECUPERACIÓN PATRIMONIAL}

La dimensión patrimonial de la intervención en Els Vilars ha ido evolucionando con el tiempo, hasta llegar a ser lo que hoy es en el marco del proyecto "Vilars 2000".

La consecuente declaración como Bé Cultural d'Interés Nacional (BCIN) en la categoría de zona arqueológica ha supuesto entrar en una tercera fase caracterizada por la redacción y ejecución del Plan Director como instrumento que asegure la realización del proyecto de acuerdo con la Llei $9 / 1993 \mathrm{del}$ Patrimoni Cultural Català (DOGC, 1807, 11.10. 1993).

En general, las tres primeras intervenciones, años 1988, 1989 y 1992, fueron actuaciones epidérmicas, con la intención de impedir la degradación y la pérdida inmediata de las estructuras afectadas. Mientras que las realizadas los años 1993 y 1995, se en- marcaron en un proyecto de recuperación, el citado Plan Trianual. La intervención más compleja tuvo lugar sobre el lado occidental del gran testigo, resto del tell original, que atraviesa de norte a sur la fortaleza. Se trataba de conservar un corte en el que se alternaban partes "blandas" (cortes estratigráficos) y partes "duras" (estructuras murarias) (Garcés, 1989: 98-107; Garcéset alii, 1993: 41-58). El gran impacto visual de los muros de hormigón hace discutible el resultado de la intervención, que deberá ser reformulada y, en cualquier caso, integrada en el Plan Director.

La definitiva confirmación de la singularidad de las estructuras constructivas, su buen estado de conservación y el excepcional interés arqueológico fue el detonante que exigió el replanteamiento de la situación y la decisión de promover la compra de las fincas. A partir de la declaración de BCIN y de la asunción del proyecto "Vilars 2000", venimos desarrollando una'estrategia de excavar-consolidar-presentar. El objetivo es doble: por un lado, rentabilizar el trabajo mostrando al público los resultados casi al mismo tiempo que se produce la excavación y haciendo evidente a la Administración el progreso experimentado; por el otro, corregir los excesos y riesgos asumidos por la priorización de la excavación extensiva y la exposición a la intemperie durante largo tiempo de restos arqueológicos desprotegidos.

Las intervenciones realizadas son modestas, teniendo en cuenta que no han de interferir ni condicionar el futuro proyecto de musealización. Hasta la fecha, han consistido en la consolidación de muros y estructuras que aparecen arrasadas por los trabajos agrícolas a una cota uniforme y se limitan, en términos generales, al recrecimiento de dos o tres hiladas para garantizar su protección. Se utiliza el mismo mampuesto y se substituye la arcilla que lo ligaba por un mortero de cal, arena y tierra del propio yacimiento. En el caso de las torres T-25, T-221 y T-247, la restauración ha supuesto la recuperación del volumen suficiente para transmitir la idea de su antigua monumentalidad (Lám. II).

Los criterios generales de intervención seguidos pueden resumirse en seis puntos: documentación, fidelidad-rigor, reversibilidad, consolidación, restauración y restitución (Alonso et alii, e.p.).

\section{LA DIFUSIÓN SOCIAL}

Desde nuestras propias convicciones hemos insistido (Junyent, 1999: 9-27) en nuestro rechazo a

T. P., 57, n. ${ }^{\circ} 2,2000$ 


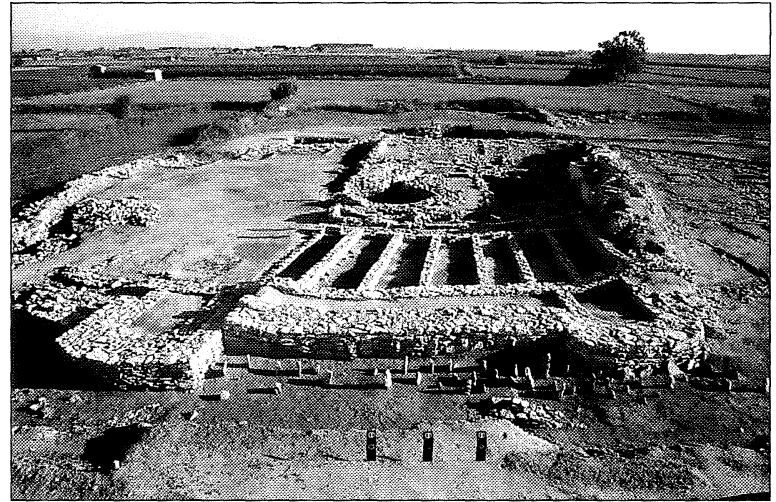

Lám. II. Vista general del barrio meridional y de la muralla de la fortaleza de Els Vilars (Arbeca, Lleida) después de los trabajos de restauración de 1999.

un sistema en el que la Administración recorta los presupuestos destinados a investigación y patrimonio, favorece las privatizaciones, envía la arqueología al mercado y obliga a los investigadores a obtener recursos como sea o a quedar en la inanidad. En este contexto, la comunicación social se instrumentaliza convirtiéndose en una forma de vender y obtener éxito y a la que el mercado dicta sus condiciones. Esta situación no debe ser aceptada acríticamente para poder combatir las perversiones inducidas (conversión de la información en mercancía, confusión de la demanda del mercado con las necesidades sociales, aceptación de los mecanismos y el lenguaje de los propios medios, competitividad, vulgarización, "impactolatría"...) y seguir reivindicando políticas públicas de investigación y patrimonio.

Para nosotros, desde la dimensión pública de la Universidad, comunicar la investigación científica universitaria es un deber social. Mediante nuestro trabajo y la comunicación de sus resultados devolvemos a la sociedad el gasto público en forma de conocimiento y de patrimonio. En realidad, asumiendo todo ésto, respondíamos al convencimiento íntimo de que los yacimientos deben ser mostrados por los arqueólogos/as y disfrutados por el público y de que, si no es así, mejor están enterrados. Al margen de estas consideraciones ético-políticas, la comunicación científica supone otros valores añadidos: mejora de la imagen de la institución en que se trabaja, promoción social e institucional del proyecto, beneficios curriculares personales, obtención de recursos y financiación y hasta satisfacción del propioego.

"Vilars 2000" tiene en la socialización de sus resultados, conocimiento y patrimonio, un objetivo prioritario. Se trata, como venimos repitiendo, de investigar un yacimiento clave para el conocimiento histórico-arqueológico, de recuperar un monumento y de hacer posible su disfrute público. A lo largo de casi quince años el proyecto se ha ido transformando hasta adquirir su actual configuración. Comenzó siendo una intervención de urgencia, se consolidó luego como proyecto de investigación y ha acabado convirtiéndose en "Vilars 2000". De forma paralela, la comunicación científica y la proyección social del proyecto se ha ido haciendo más sistemática y hoy podemos afirmar, que constituye una de las líneas estratégicas y que, como tal, ha resultado básica en la consolidación y desarrollo del mismo. Sus actuales perspectivas son ciertamente inimaginables sin el grado de presencia social y mediática alcanzado.

El equipo, con todos los problemas derivados de la falta de medios, tiempo y profesionalidad en estas lides, ha dedicado su esfuerzo a conseguirlo. A diferencia del celo protector con que, hace tan sólo unos años, investigadores e investigadoras envolvíamos los resultados del trabajo antes de ser publicados, nuestra estrategia ha sido literalmente la contraria, entendiendo que toda difusión de los mismos promocionaba el proyecto. Buen ejemplo de lo que decimos es la aparición de restituciones, incluso, de la propia planta de la fortaleza antes de su publicación científica por parte del equipo. Entre las acciones y actividades de difusión desarrolladas, subrayamos:

1. Participación en campañas institucionales y asistencia a reuniones sobre la comunicación científica, por ejemplo, La Recerca, innovació $i$ servei (Sitges, 13-14 de noviembre de 1997) y Comuniquem la Ciència (Lleida, 11 de febrero de 1998), organizada por la Universitat de Lleida.

2. Estrategia tendente a garantizar la presencia en:

- Prensa. Colaboración con el Gabinete de Prensa de la Universitat de Lleida. Presencia regular en la prensa de ámbito nacional (El País, Avui, La Vanguardia, El Periódico) y continuada en la prensa local (Segre y La Mañana).

- Radio y Televisión. Grabación y emisión (13 y 15 de octubre de 1998) por parte del Canal 33, de un capítulo de Més Enllà del 2000, el programa de divulgación científica de mayor prestigio de Televisió de Catalunya. Grabación y emisión de un ca- 


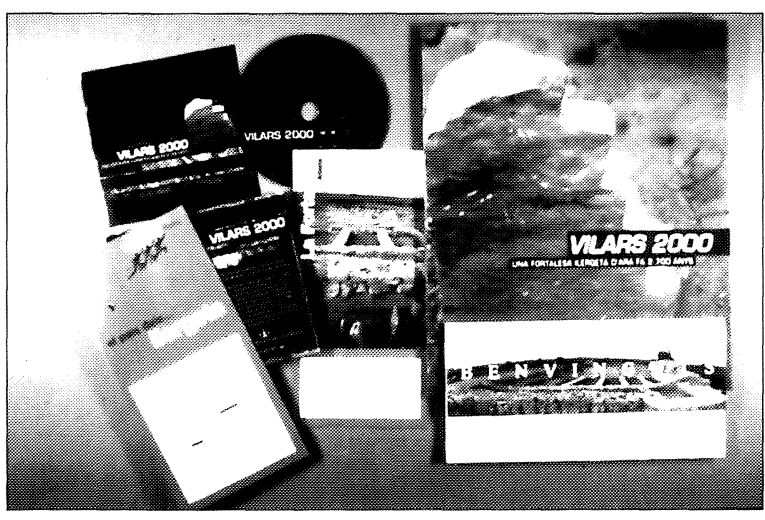

Lám. III. Edición de materiales de difusión del proyecto "Vilars 2000".

pítulo de Catalunya Retalls, programa de Televisión Española de Catalunya. Grabación de un programa de unos 15' de duración para Canal Digital sobre realidad virtual y arqueología (1999). Grabación (mayo 2000) para una serie de TV3 de historia de Catalunya. Participación en diferentes programas culturales y de información general de radio, como L'Observatori de Radio Nacional de España en Barcelona, L'Aparador de Catalunya Ràdio o La Tertúlia" de ComRàdio Lleida, etc...

3. Edición de materiales de difusión (Lám. III):

- Libro. I. Garcés et alii, Vilars 2000. Una fortaleza ilergeta d'ara fa 2700 anys, Ajuntament d'Arbeca, Fundació 700Aniversari, Universitat de Lleida, Lleida 1997. Texto en cuatro idiomas: catalán, castellano, francés e inglés.

- CD-ROM. Vilars 2000. Edición electrónica del libro mencionado. Incluye el vídeo Vilars Virtual. Barcelona, 1999.

- Vídeo. El primer producto del proyecto "Vilars Virtual" ha sido un vídeo de edición limitada y uso casi exclusivamente promocional que se ha enviado a instituciones y potenciales patrocinadores. En su formato actual de 6' (versión 1998), puede verse en el web site, en el CD-ROM o en el propio Ajuntament d'Arbeca tras una visita a la fortaleza. Está previsto disponer en diciembre de 2000 de una nueva versión centrada en las fases Vilars 0 y I, de unos 20' de duración y que incorporará la paleotopografía y el paisaje vegetal del entorno de la fortaleza. Así mismo, en el recorrido interior se podrá penetrar en una vivienda. Los primeros resultados han sido avanzados en el 28 th International Congress CAA, Ljubljana (Eslovenia), abril de 2000.
4. Difusión escolar y edición de materiales didácticos:

A lo largo del mes de mayo de 2000 se ha desarrollado en colaboración con el Institut de Ciències de l'Educació de la Universitat de Lleida la actividad "Vilars ens descobreix la cultura ibèrica". La experiencia ha tenido un carácter experimental y ha servido para poner a prueba talleres y materiales didácticos. Han participado más de un centenar de niños y niñas del Ciclo Superior de Primaria de las escuelas CEIPAlbirka (Arbeca), CEIP Joan XXIII (les Borges Blanques) y ZER Riu Corb (Belianes). Tres monitores/as han organizado una sesión previa en las escuelas, cuatro talleres sobre el terreno (descubrimiento de la fortaleza, urbanismo, mundo funerario, el trabajo de los arqueólogos: la máquina de flotación) y una sesión de reflexión y conclusión. A partir del próximo año se formalizará la oferta a todas las escuelas leridanas, adaptándolas también a alumnado de primer Ciclo de ESO, y se editarán los materiales.

Asimismo, hemos colaborado con la Editorial Baula y el Museu d'Arqueologia de Catalunya en la preparación de La ruta dels ibers. Crédit variable d'ampliació, Ciències Socials, Educació Secundària Obligatòria (Hernàndez et alii, 1999) y en una experiencia similar con la Fundació Enciclopèdia Catalana; en ambos proyectos tiene una presencia significada la fortaleza arbequina.

\section{Presencia en Internet:}

Desde febrero de 1999 el proyecto dispone de un web site en la red con dominio propio: http:// www.vilars2000.com. El internauta puede acceder al contenido del libro Vilars 2000. Una fortalesa d'ara fa 2700 anys, a un vídeo presentando una restitución virtual, la bibliografía científica, el estado actualizado del proyecto, enlaces con webs sobre la cultura ibérica, información turística complementaria y, finalmente, información sobre las actividades previstas (excavaciones, cursos, publicaciones, presencia en reuniones, etc.). Un buzón permite conectar con el correo electrónico de los miembros del equipo investigador.

\section{6. "La Ruta dels Ibers":}

La fortaleza de Els Vilars de Arbeca se incorporó en junio del pasado año a "La Ruta dels Ibers". Se trata de un proyecto del Museu d'Arqueologia de Catalunya con el patrocinio inicial de la Fundació "La Caixa" que pretende poner al alcance del público los yacimientos ibéricos más relevantes del 
país, aprovechando la sensibilización suscitada por la exposición "Los Iberos. Príncipes de Occidente" (París, octubre 1997-enero 1998; Barcelona, eneroabril 1998; Bonn, mayo-agosto 1998) e intentando materializarla en una serie de acciones dinamizadoras en los mismos.

Para la fortaleza arbequina la incorporación a "La Ruta dels Ibers" ha supuesto un importante primer paso en su presentación al público con la instalación de una infraestructura de acogida (elementos de señalización en la carretera y accesos, puntos de información y paneles informativos), la edición de material (folletos promocionales de ruta y yacimiento y tríptico de la fortaleza) y, sobre todo, la promoción desde el Museu d'Arqueologia de Catalunya y sus diferentes sedes (Lám. IV). El proyecto y las espectativas por él creadas ganan credibilidad y es de esperar que con la incorporación a la ruta aumenten el número de visitantes y también la atención prestada por las instituciones y los patrocinadores.

7. Incorporación a la oferta del Patronat de Turisme de la Diputació de Lleida.

Aparición en el catálogo de paquetes turísticos "Gaudir Lleida 2000".

\section{Inserción en el tejido social.}

Nuestra estrategia de dar a conocer el proyecto, entendiendo que su justificación última está en su socialización y que ésta es, además, la única vía posible para demostrar su interés y rentabilidad cultural y socioeconómica a las instituciones que deben asumirlo, ha tenido y tiene un objetivo prioritario en su inserción en su entorno social. Conseguir que la población conozca y estime, y se sienta-ipor qué no?- orgullosa de poseer en su término municipal unos restos arqueológicos excepcionales, que entienda que la recuperación de la fortaleza puede contribuir a mejorar la vida de los ciudadanos y las ciudadanas, a hacer un pueblo más próspero. La Arbeca de hoy y del futuro ha de desarrollar, al lado de las actividades económicas tradicionales, nuevas formas de riqueza, potenciar los recursos culturales, históricos, etnográficos y naturales y aprovechar la singularidad de su oferta ante las nuevas formas del turismo y del ocio. Únicamente en la medida que la población asuma este mensaje, un consistorio de posibilidades económicas limitadas estará en condiciones de destinar parte de sus presupuestos a la recuperación y mantenimiento de la fortaleza.

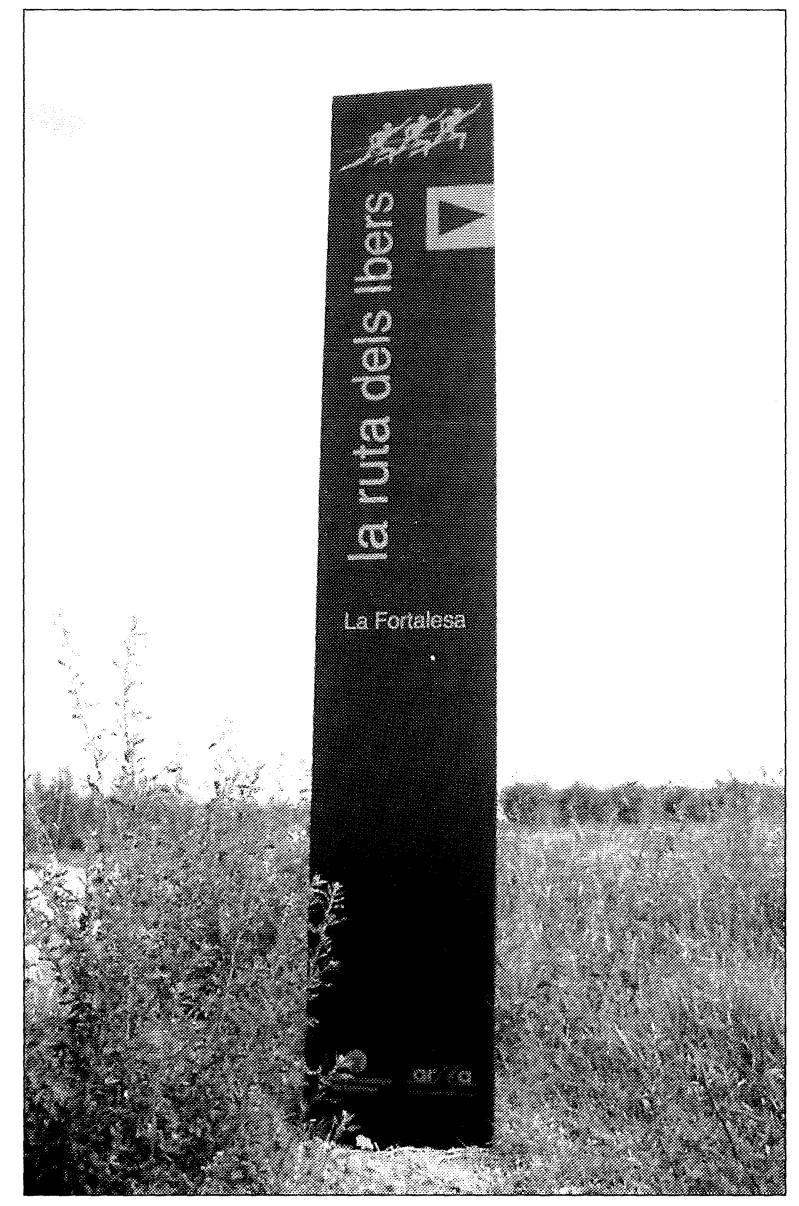

Lám. IV. Señalización de acceso de la Ruta dels Ibers.

Desde el inicio de las excavaciones y especialmente estos últimos años, hemos dedicado una especial atención a conseguir situar el proyecto en el centro de la vida cultural de la población y lograr la máxima comprensión e implicación en el mismo. Para ello, el equipo ha prestado atención preferente a la escuela y los niños y niñas han contribuido en gran medida a dar a conocer e interesar a sus familias en el yacimiento; ha organizado jornadas de puertas abiertas todas las campañas de excavación y ha impartido conferencias y participado en Jornadas Culturales. La última jornada de puertas abiertas (30 de septiembre y 1 de octubre de 2000), celebrada conjuntamente con las Jornadas Europeas del Patrimonio promovidas por el Consejo de Europa, incluía una visita desde el aire mediante un globo cautivo y ha supuesto la participación de más de cuatrocientas personas. En la actualidad, estudiamos la posibilidad de constituir una "Asociación de Amigos de Els Vilars". Un hecho que, desde esta 
perspectiva, no consideramos anecdótico, pues muestra el grado de presencia e implicación del equipo de arqueólogos/as en la población, fue que uno de sus miembros, E. Junyent, pronunciase el pregón de la Festa Major (agosto de 1998).

Este esfuerzo por difundir los resultados conseguidos y, al tiempo, promocionar el proyecto, ha dado, globalmente considerados, unos frutos espléndidos. Tanto, que en estos momentos corremos el riesgo de defraudar al visitante en las expectativas generadas, al haber sobrepasado la realidad de la excavación y la consolidación de la fortaleza, en parte por no haberse concretado determinadas iniciativas, en particular, la creación de la proyectada Escuela-Taller o el Plan de Ocupación, y, sobre todo, debido a la falta de recursos y al retraso en la redacción de los documentos técnicos que han de materializar el Plan Director.

\section{EL DESARROLLO DEL PLAN DIRECTOR}

El objetivo global del "Plan Director Vilars 2000 ", concebido como instrumento de planificación y gestión, es ofrecer a la sociedad del siglo XXI $y$ al futuro una fortaleza ilergeta excavada, restaurada y parcialmente reconstruída y ambientada, un monumento visitable, dotado de los servicios necesarios para hacer de él una realidad social y cultural viva y un proyecto de investigación activo vinculado a la Universitat de Lleida. De la misma manera ha de constituir un motor de dinamización económica y cultural para las comarcas occidentales de Catalunya, convirtiéndose en una referencia obligada del turismo cultural en nuestra tierra y en un punto de apoyo básico de la enseñanza y la difusión de nuestra historia. Sus líneas generales han sido anteriormente expuestas (Garcés et alii, 1997) y recientemente presentadas en el I Encuentro Museos, Arqueología y Turismo, organizado por el Museo Arqueológico Nacional (Madrid, 1999) (Alonsoet alii, e.p.). La Direcció General del Patrimoni Cultural de la Generalitat de Catalunya nos ha encargado, finalmente, la redacción del Plan Director y sus documentos técnicos 1 y 2 .

En estos momentos trabajamos con la siguiente propuesta de estructuración y calendario:

Primera fase: 2000-2002. Investigación arqueológica y planeamiento.

Segunda fase: 2000-2003. Intervención y consolidación.
Tercera fase: 2004. Musealización.

Los objetivos, metodología y programa de trabajos de las dos primeras se formulan respectivamente en los documentos: 1. "Proyecto de Excavaciones Arqueológicas, Investigación y Documentación" y 2. "Plan Especial de Protección Arqueológica y Ordenación de Els Vilars. Proyecto de Consolidación y Restauración".

\section{IMPLICACIÓN INSTITUCIONAL, GESTIÓN Y RECURSOS}

Hacer realidad "Vilars 2000" exige una investigación solvente, pero ello no ha resultado suficiente. Tampoco lo ha sido, hasta la fecha, mostrar la potencial monumentalidad del conjunto. Debemos demostrar su condición de "pozo de petróleo", de fuente de riqueza, de factor de desarrollo económico, para de este modo poder generar una dinámica capaz de proporcionar los recursos necesarios para investigar, recuperar y socializar el patrimonio.

Había que hacer camino andando y mostrar la rentabilidad de los resultados que se iban obteniendo, como forma de sensibilizar a las distintas administraciones y posibles patrocinadores. En este terreno hemos dado los primeros pasos y "Vilars 2000" cuenta ya con el patrocinio de las primeras empresas, Prefabricats Pujol S.A. y SCCL del Camp l'Arbequina. Pretendemos ofrecer algo así como un patronazgo "a la carta": al patrocinador se le presentan las diferentes opciones, por ejemplo, la consolidación y restauración de una torre determinada, la puerta Este, etc., la investigación de tal o cual sector o tema, los talleres escolares, el nuevo vídeo, el mantenimiento de laweb, una publicación monográfica, una reunión científica internacional, etc. El objetivo es que el patrocinador sepa en qué se va a gastar su dinero y su inversión luzca individualizada en vez de ir a parar anónimamente a un pozo sin fondo. Pero la procedencia de los recursos aplicados es básicamente pública: Ajuntament d'Arbeca, Direcció General del Patrimoni Cultural (Servei d'Arqueologia), Diputació de Lleida (Institut d'Estudis Ilerdencs y Patronat de Promoció Econòmica de les Terres de Lleida) y Ministerio de Educación y Cultura. Sin embargo, su cuantía y su carácter irregular y discontinuo vía subvenciones impiden planificar inversiones plurianuales y programar con la necesaria continuidad. Hay que implicar a los grupos de acción local y a las instituciones para poder acceder a los únicos presupuestos, 
hoy en día, asequibles y destinados a este tipo de iniciativas: el $1 \%$ cultural, administrado por el Ministerio de Fomento y la Generalitat de Catalunya, la actuación comunitaria en el ámbito cultural, programa Cultura 2000 (2000-2004), y los fondos estructurales y de cohesión europeos aplicados a la política comunitaria de desarrollo rural (Política Agraria Común 2000-2006), cuyo enfoque multisectorial e integrado de la economía rural les hace adecuados a este tipo de proyectos. Es el caso del Programa de Desenvolupament Rural de Catalunya (PDR), resultado de la aplicación de las previsiones de la Agenda 2000 en el ámbito de la agricultura y el medio rural y que pretende la dinamización mediante actividades complementarias o alternativas a la agricultura, y de la iniciativa Leader+ de la Unión Europea.

Por ello, junto a la redacción en curso de los documentos técnicos del Plan Director, el objetivo estratégico prioritario es la constitución del $\mathrm{Pa}$ tronat d'Arqueologia de la Fortalesa d'Arbeca. El patronato, constituido por elAjuntament d'Arbeca, la Direcció General del Patrimoni Cultural, la Diputació de Lleida, el Consell Comarcal de les Garrigues y la Universitat de Lleida, deberá garantizar el protagonismo de las instituciones agrupadas en consorcio y procurar la obtención y gestión de los recursos necesarios.

En cuanto al modelo de gestión futura del yacimiento musealizado y visitable, pasa por su integración, teniendo en cuenta que se trata de una propiedad municipal, como sección o entidad vinculada al despliegue territorial del Museu d'Arqueologia de Catalunya que, como es sabido, tiene sedes en Girona, Empúries, Ullastret y Olèrdola, según las previsiones del propio Departament de Cultura, anunciadas por el Conseller de Cultura ante el Parlamento de Catalunya (Notes per a la substantació de la sessió informativa del Conseller de Cultura, sobre les línies d'actuació del Govern en matèria de Cultura i Esport (NT 355-00004/06, 23 de març 2000, pàg. 53).

\section{BIBLIOGRAFÍA}

AA.VV. (1995): "Datació radiocarbònica i calibratge". Revista d'Arqueologia de Ponent, 5: 249-275.

Agustí, B.; Alonso, N.; Garcés, I.; Junyent, E.; LafuenTE, A. y LÓPEZ, J.B. (2000): "Una inhumación múltiple de perinatales en la fortaleza de Els Vilars (Arbeca, Lleida) y las prácticas de enterramiento en hábitat durante la I Edad del Hierro en el valle del Segre (Cataluña)”.
Monographies d'Archéologie Méditerranéenne, 5: 305-324.

Alonso, N. (1999): De la llavor a la farina. Els processos agrícoles protohistòrics a la Catalunya occidental. Monographies d'Archéologie Méditerranéenne, 4. Lattes.

Alonso, N.; Garcés, I.; Junyent, E.; Lafuente, A.; LóPez, J.B.; Mrró, J.M.; Ros, M.T. y Rovira, C. (1996): "L'assentament de Els Vilars (Arbeca, les Garrigues): Territori, recursos i activitats productives". Gala, 3-5: 319-339.

Alonso, N.; Junyent, E.; Lafuente, A. y López, J.B. (1998): "Poder, símbolo y territorio: el caso de la fortaleza de Arbeca". Congreso Internacional: Els Ibers, princeps d'Occident (Barcelona, 1998): 355-372. Barcelona.

- (1999): “Chronométrie de l’Âge des Métaux dans la basse vallée du Segre(Catalogne, Espagne) à partir des datations C14".Actes du 3ème Colloque International C14 et Archéologie (Lyon, 1998). Mémoires de la Société Préhistorique Française, XXVI: 287-292.

Alonso, N.; MuÑoz, D.; Junyent, E.; Lafuente, A.; LóPez, J.B.; LORÉs, J.; PÉREZ, M.; y TARTERA, E. (2000): “Virtual reality as an extension of the archaeological record: reconstruction of the iron age fortress els Vilars (Arbeca, Catalonia, Spain)". En J.A. Barceló, M. Forte y D.H. Sanders (eds.): Virtual Reality in Archaeology, BAR International Series 843. Oxford: 225-231.

Alonso, N.; JunYent, E.; LAfuente, A. y LóPez, J.B. y TARTERA, E. (en prensa): "El proyecto Vilars 2000". I Encuentro Museos, Arqueología y Turismo. Museo Arqueológico Nacional (Madrid, 1999).

Baillie, M.G.L. y Pilcher, J.R. (1983): "Some observations on the High-Precision Calibration of Routine Dates". En B.S. Ottaway (ed.): Archaeology, Dendrochronology and the Radiocarbon Calibration Curve. Department of Archaeology Occasional Paper, 9. University of Edinburgh: 51-63.

CAstro, P.V.; Lull, V. y Micó, R. (1996): Cronología de la Prehistoria Reciente de la Península Ibérica y Baleares (c.2800-900 cal ANE). BAR International Series, 652. Oxford.

GARCÉs, I. (1989): “Avanç de les excavacions al poblat ibèric delsVilars d'Arbeca (Les Garrigues)". Excavacions arqueològiques d'urgència a les comarques de Lleida. Excavacions arqueològiques a Catalunya, 9: 98-107.

Garcés, I.; JunYent, E.; LAFuente, A. y LóPEZ, J.B. (1991): "Sistema de registro y tratamiento automático de la información en el yacimiento protohistórico de Els Vilars (Arbeca, Lleida)". Complutum, 1: 189-210.

- (1997): Vilars 2000. Una fortalesa ilergeta d'ara fa 2700 anys. Ajuntament d'Arbeca i Universitat de Lleida. Lleida.

- (2000): "L'habitat dans la moyenne et basse vallée du Segre au 1er. Age du fer. Le cas du village fortifié de Els Vilars (Arbeca, Lleida)". En J. Gascó y F. Claustre

T. P., 57, n. ${ }^{\circ} 2,2000$ 
(eds.): Habitats, économies et sociétés du Nord-Ouest méditerranéen de l'Âge du Bronze au premier Âge du Fer. Actes du XXV Congrès Préhistorique de France (Carcasonne, 1994): 147-157. Paris.

Hernández, X.; Prats, J. y Santacana, J. (1999): La ruta dels ibers. Crèdit variable d'ampliació, Ciències Socials, Educació Secundària Obligatòria. Edicions Baula i Museu d'Arqueologia de Catalunya. Barcelona.

JAMES, P. (1993): Siglos de oscuridad. Desafío a la cronología tradicional del mundo antiguo. Ed. Crítica. Barcelona.
Junyent, E. (1982): “Tossal del Molinet, El Poal”. Les Excavacions Arqueològiques a Catalunya en els darrers anys. Excavacions Arqueològiques a Catalunya, 1: 256-257.

- (1999): "Patrimoni arqueològic, difusió i mercat: algunes reflexions". Cota Zero, 15: 9-27.

LóPEZ, J.B. (1999): "Els temps prehistòrics i antics (fins al segle Vè)". En B. De Riquer (dir.): Cronologia dels Països Catalans. Història, societat, economia, cultura i ciència. Ed. Pòrtic. Barcelona: 11-19.

OlsEN, O. (1980): "Rabies Archaeologorum". Antiquity, 54: 15-20. 